\title{
Early-onset obesity and the unwanted promise of thrombosis
}

\author{
Richard C. Becker
}

Published online: 25 February 2011

(C) Springer Science+Business Media, LLC 2011

Obesity is rapidly becoming a global epidemic of unparalleled proportions. While the initial alarm was sounded by the National Center for Health Statistics in 1994 (reviewed in Ref. [1]), changes in body mass index have accelerated rapidly in the past 5 years among children, adolescents and adults. In addition to its association with several atherogenic states, including type 2 diabetes mellitus, systemic hypertension and dyslipidemia, obesity is an independent and strong risk factor for thrombosis as a proximate cause of stroke, myocardial infarction and venous thromboembolism [2, 3]. Data from the National Hospital Discharge Survey support a 2 to 3 -fold increased risk for both obese men and women, particularly in those who are less than 40 years of age [4]. With obesity comes the unwanted promise of thrombosis among an entire generation of people with childhood and adolescent (early) onset obesity.

\section{Obesity as a systemic disease}

Beyond traditional risk factors for atherothrombosis observed frequently among obese individuals, one most consider additional acquired metabolic abnormalities and contributing disease states [5,6]. Accumulating evidence strongly supports that obesity is a systemic disease characterized by low-grade inflammation which in turn, leads to insulin resistance-both are associated strongly with systemic markers of inflammation [7] and atherosclerosis. Adipose tissue itself is composed of several cell types, including lipid-laden mature adipocytes, vascular stromal

R. C. Becker $(\square)$

Duke Clinical Research Institute, Duke University School of Medicine, Durham, USA

e-mail: becke021@mc.duke.edu cells and macrophages. Chronic, low-grade inflammation is a characteristic of obesity and adipocytes have been shown to produce TNF- $\alpha$. In addition, stromovascular matrixcontaining immune cells are also rich source of TNF- $\alpha$ [8]. Co-culture of differentiated 3T3-L1 adipocytes and macrophages upregulate proinflammatory cytokines such as TNF- $\alpha$ and down-regulate the anti-inflammatory and antiatherothrombotic cytokine adiponectin [9]. These inflammatory changes are augmented by adipose vascular stroma fraction, suggesting that inflammatory mediators participate in a maladaptive "paracrine loop" between adipocytes and macrophages.

Inflammation-sensitive plasma proteins, including fibrinogen, orosomucoid, $\alpha 1$-antitrypsin, haptoglobin and ceruloplasmin increase steadily with body mass index, and correlate with cardiovascular death, MI and stroke [10]. Soluble CD-40L, a marker of inflammation, platelet activation and prothrombotic potential, is elevated among obese individuals, declining along with fasting insulin, MCP-1 and hs-CRP levels after bariatric surgery-associated weight loss [11]. Similarly, circulating procoagulant microparticles have been documented in obesity, with levels 3 to 4 -fold higher than age-matched, non-obese controls [12].

Abdominal fat-related activation of the innate and adaptive immune systems has been implicated as a contributor of cardiovascular disease in obese individuals. Systemic adaptive immune activation is impacted and regulated by innate immune system activation [13].

Dysfunctional adipose tissue may represent an important link between obesity and metabolic disorders. In particular, preadipocyte factor 1 (Pref-1) has been shown to influence adipocyte size and functionality. A study of patients undergoing bariatric surgery identified lower levels of Pref1 in both subcutaneous and omental fat tissue samples in 


\section{Evolutionary \\ Maladaption \\ Disease \\ Response}

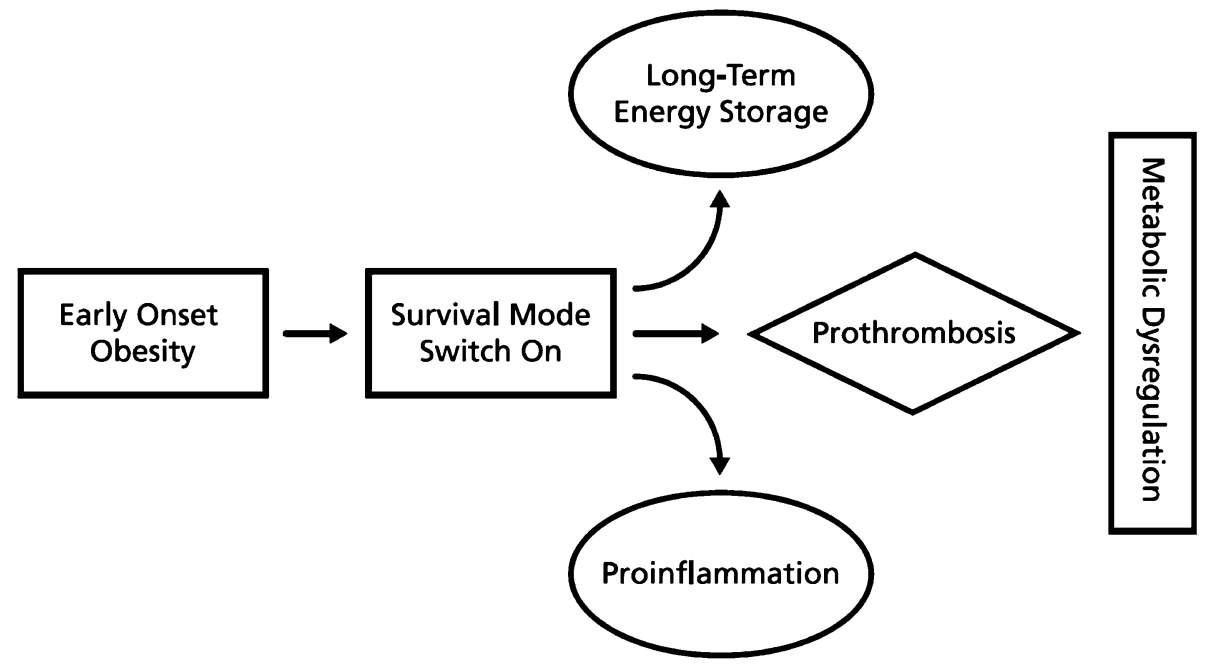

Fig. 1 The natural history of obesity may initially represent an evolutionary response to caloric intake beyond ordinary requirements, assuming a survival mode and energy storage, primed hemostasis and natural defense to infection and other "non-self" offenders. A second stage of obesity represents the point of maladaption, wherein compensatory mechanisms and preparation for a major challenge to

the group classified as metabolically healthy compared to those with metabolic abnormalities. Omental Pref-1 correlated with adipocyte size, percent fatty liver, fasting glucose, triglyceride and adiponectin [14]. Dysregulated production and secretion of adipokines from dysfunctional adipose tissue likely contributes to the systemic metabolic abnormalities observed in obesity (reviewed in [15]) (Fig. 1).

Obesity, cardiovascular disease and thrombosis

Body mass index (the weight in kilograms divided by the height in meters) is associated with increased all-cause mortality and mortality from cardiovascular disease, with the greatest overall risk for adults (ages 19-84 years) in the 35.0-39.9 (HR 1.88) and 40.0-49.9 (HR2.51) categories [16]. Obesity has been associated with increased visceral fat, including non-alcoholic fatty liver damage. In this disorder, coagulation factors VIII, IX, XI and XII-related activities are increased independent of age and gender [17]. In addition, obesity is a risk factor for chronic venous insufficiency and venous thromboembolism. Individuals with a BMI $>30 \mathrm{~kg} / \mathrm{m}^{2}$ have decreased lower extremity venous peak velocity, mean velocity, velocity amplitudes (peak velocity-minimum velocity) and shear stress [18]. Overall obese individuals are 2-fold more likely to experience venous thromboembolism than non-obese individuals, particularly if obesity is accompanied by decreased the host exceeds natural environmental evolutionary pressures. The development of insulin resistance, diabetes and hypertension signal the onset of metabolic dysregulation and disease propensity, including atherosclerotic vascular disease and venous thromboembolic disease. Early-onset obesity may be more likely to invoke a "survival switch" and a robust maladaptive response than later onset obesity

muscle strength [19] or inherited thrombophilias, including the factor $\mathrm{V}$ Leiden gene [20] and prothrombin gene mutations [21].

The mammalian silent information regulator-two 1 (Sirt1) attenuates the potentially injurious effects of obesity on vascular endothelial cells. In animal models, Sirt1 inhibition by splitomycin or sirtinol augment cytokineinduced endothelial tissue factor expression as well as surface activity. Sirt1 inhibition is also associated with TF mRNA expression, TF promoter activity, nuclear translocation and DNA binding of the p65 subunit of $\mathrm{NF}_{\mathrm{k}} \mathrm{B}\left(\mathrm{NF}_{\mathrm{k}} \mathrm{B} /\right.$ p65) [22]. In vivo, mice treated with the Sirt1 inhibitor splitomycin exhibit enhanced TF activity within vessel walls and accelerated carotid artery thrombus formation in a photochemical injury model. Accordingly, abnormalities at the molecular or protein level of Sirt1 in obese individuals could contribute to a thrombotic phenotype.

\section{Genetics of obesity}

Family-based linkage analysis and candidate-gene studies have been useful to identify genes responsible for extreme forms of early-onset disease segregating as single gene (Mendelian) disorders, including mutations in genes encoding leptin, the leptin receptor, and proopiomelanocortin. 
Genome-wide association studies of population-based samples to examine the full range of BMI values have identified 30 loci influencing BMI and the risk of obesity. The strongest to date is an association with variants within fat mass and obesity-related gene (FTO). Other signals near BDNF, SH2B1 and NEGR1 reinforce a possibility that obesity is a disorder related to hypothalamic function. Genome wide analyses of patterns of fat distribution have characterized 15 loci which are largely distinct from those influencing overall adiposity. More recent association analyses of nearly 250,000 individuals [23] within the Genetic Investigation of Anthropometric Traits (TRAITS) consortium revealed six new loci near GPRc5B, MAP2K5LBXCOR1, TNN13K, LRRN6C, FLJ34779-HMGCR and PRKD1 associated with BMI or other obesity-related traits [23]. The BMI-increasing allele showed directionally consistent effects on body fat percentage. In addition, the BMI SNPs were in linkage disequilibrium with common missense SNPs or copy number variants.

Genome-wide association studies in adults have revealed obesity-related genes that also explain BMI variation in children [24] and adolescents [25]. Environmental conditions can influence transgenerational gene expression via reversible heritable epigenetic mechanisms. Epigenetic changes in DNA methylation patterns at $\mathrm{CpG}$ sites (epimutations) or corrupt chromatin states of key inflammatory genes and noncoding RNAs may influence chronic inflammatory disorders, including obesity [26]. In the Framingham offspring cohort [27] genetic variance clustered downstream from LYPLAL-1, which encodes a lipase/esterase expressed in adipose tissue showed a consistent BMI association in obese women, but not among men.

It must be acknowledged, however, that the common variants associated with obesity account for less than $1 \%$ of the overall variance in BMI, supporting ongoing investigation to better characterize functional signatures to include downstream consequences of variants lying outside of the genes coding region (exome).

Obesity is, for the most part an acquired, global epidemic of unparalleled proportion, affecting tens of millions of people world-wide. Early-onset obesity, developing during childhood and adolescence is particularly problematic as it introduces hypertension, diabetes mellitus and atherosclerotic vascular disease at an uncharacteristically premature stage in life. A chronic inflammatory and altered metabolic state contribute to the onset of vascular disease, and in addition contribute to the development of a highly prothrombotic condition. The unwanted promise of thrombosis for people around the world represents an immediate call to action for the public health, medical and scientific communities.

\section{References}

1. Poirier P et al (2006) Obesity and cardiovascular disease: pathophysiology, evaluation, and effect of weight loss: an update of the 1997 American heart association scientific statement on obesity and heart disease from the Obesity Committee of the Council on Nutrition, Physical Activity, and Metabolism. Circulation 113(6):898-918

2. Goldhaber SZ et al (1997) A prospective study of risk factors for pulmonary embolism in women. JAMA 277(8):642-645

3. Blaszyk H et al (1999) Death from pulmonary thromboembolism in severe obesity: lack of association with established genetic and clinical risk factors. Virchows Arch 434(6):529-532

4. Stein PD, Beemath A, Olson RE (2005) Obesity as a risk factor in venous thromboembolism. Am J Med 118(9):978-980

5. Poirier P et al (2006) Obesity and cardiovascular disease: pathophysiology, evaluation, and effect of weight loss. Arterioscler Thromb Vasc Biol 26(5):968-976

6. Becker RC (2010) A clinical cardiology perspective of thrombophilias. J Thromb Thrombolys 30(3):378-389

7. Grignani G, Maiolo A (2000) Cytokines and hemostasis. Haematologica 85(9):967-972

8. Red Eagle A, Chawla A (2010) In obesity and weight loss, all roads lead to the mighty macrophage. J Clin Invest 120(10): $3437-3440$

9. Suganami T, Nishida J, Ogawa Y (2005) A paracrine loop between adipocytes and macrophages aggravates inflammatory changes: role of free fatty acids and tumor necrosis factor alpha. Arterioscler Thromb Vasc Biol 25(10):2062-2068

10. Engstrom $\mathrm{G}$ et al (2004) Incidence of obesity-associated cardiovascular disease is related to inflammation-sensitive plasma proteins: a population-based cohort study. Arterioscler Thromb Vasc Biol 24(8):1498-1502

11. Schernthaner GH et al (2006) Soluble CD40L in patients with morbid obesity: significant reduction after bariatric surgery. Eur J Clin Invest 36(6):395-401

12. Goichot B et al (2006) Circulating procoagulant microparticles in obesity. Diabetes Metab 32(1):82-85

13. Thewissen MM et al (2011) Abdominal fat mass is associated with adaptive immune activation: the CODAM study. Obesity

14. O'Connell J et al. (2011) Preadipocyte Factor-1 is associated with metabolic profile in severe obesity. J Clin Endocrinol Metab

15. Ouchi $\mathrm{N}$ et al. (2011) Adipokines in inflammation and metabolic disease. Nat Rev Immunol 11(2):85-97

16. Berrington de Gonzalez A et al (2010) Body-mass index and mortality among 1.46 million white adults. N Engl J Med 363(23):2211-2219

17. Kotronen A et al (2011) Increased coagulation factor VIII, IX, XI and XII activities in non-alcoholic fatty liver disease. Liver Int Off J Int Assoc Study Liver 31(2):176-183

18. Willenberg $T$ et al (2010) Impact of obesity on venous hemodynamics of the lower limbs. J Vasc Surg 52(3):664-668

19. Di Nisio, M., et al (2010) Obesity, poor muscle strength, and venous thromboembolism in older persons: the InCHIANTI study. J Gerontol A Biol Sci Med Sci

20. Cushman M et al (2010) Risk factors for peripheral venous disease resemble those for venous thrombosis: the san diego population study. J Thromb Haemost 8(8):1730-1735

21. Severinsen MT et al (2010) Genetic susceptibility, smoking, obesity and risk of venous thromboembolism. Br J Haematol 149(2):273-279

22. Breitenstein A et al (2011) Sirt1 inhibition promotes in vivo arterial thrombosis and tissue factor expression in stimulated cells. Cardiovasc Res 89(2):464-472 
23. Speliotes EK et al (2010) Association analyses of 249, 796 individuals reveal 18 new loci associated with body mass index. Nat Genet 42(11):937-948

24. Wu L et al (2010) Associations of six single nucleotide polymorphisms in obesity-related genes with BMI and risk of obesity in Chinese children. Diabetes 59(12):3085-3089

25. den Hoed M et al (2010) Genetic susceptibility to obesity and related traits in childhood and adolescence: influence of loci identified by genome-wide association studies. Diabetes 59(11): 2980-2988

26. vel Szic KS et al (2010) Nature or nurture: let food be your epigenetic medicine in chronic inflammatory disorders. Biochem Pharmacol 80(12):1816-1832

27. Benjamin AM et al. (2011) Gene by sex interaction for measures of obesity in the framingham heart study. J Obes 2011: p. 329038 